\title{
Pregnant women admitted with urinary tract infections to a public sector hospital in South Africa: Are there lessons to learn?
}

\author{
Francois de Paul Siemefo Kamganga*, Hopolang C Maise ${ }^{a}$ and Jagidesa Moodley ${ }^{b}$ \\ ${ }^{a}$ Department of Obstetrics and Gynaecology and Women's Health and HIV Research Group, University of KwaZulu-Natal, Durban, South Africa \\ ${ }^{b}$ Women's Health and HIV Research Group, University of KwaZulu-Natal, Durban, South Africa \\ *Corresponding author, email: siemefo1@yahoo.fr
}

\begin{abstract}
Background: Urinary tract infection (UTI) is associated with poor maternal and foetal outcomes. There is little information on UTI in pregnancy in South Africa.

Objectives: To evaluate the frequency of UTI admissions of pregnant women admitted to a public health facility; and, to describe the outcomes of pregnancies complicated by UTI in our study population.

Methods: A retrospective chart review of pregnant women admitted with the diagnosis of UTI during the period of 1 January 2012 to 31 December 2012 was conducted. A midstream urine sample of women admitted with symptoms suggestive of UTI was collected for culture. The diagnosis was confirmed if the culture was positive. The data was analysed using SPSS version 21 and descriptive statistics, viz. percentages, frequency and means were estimated.

Results: Of 9,881 admissions, 494 (5\%) had a diagnosis of UTI based on clinical features. Sixty had positive cultures and were confirmed as having UTI. Women with UTI had high rates of preterm ruptured membranes $(n=5,8.3 \%)$, preterm deliveries ( $n=19,31.6 \%)$, anaemia $(n=26,43.3 \%)$ and renal impairment $(n=4,6.6 \%)$. Two cases of pyelonephritis required admission to an ICU because they developed acute respiratory distress syndrome. The most common pathogen isolated using cultures was Escherichia coli $(n=24,40 \%)$. Only $10 \%$ had repeat urine cultures.

Conclusion: UTI represents $5 \%$ of admissions at the study site. This study highlights the need to improve the quality of care of pregnant women with UTI.
\end{abstract}

Keywords: obstetric complications, positive urine cultures, pregnancy, urinary tract infections

\section{Introduction}

Urinary tract infection (UTI) is one of the most common medical complications in pregnancy. ${ }^{1-3}$ In the majority of cases, UTIs are preceded by asymptomatic bacteriuria. ${ }^{1-3}$ If untreated in pregnancy, $20 \%$ to $30 \%$ go on to develop acute pyelonephritis. ${ }^{3,4}$ Acute pyelonephritis carries significant risks in pregnancy including: septicaemia, anaemia, transient renal dysfunction, preterm labour, intrauterine growth restriction (IUGR), premature rupture of membranes and preeclampsia. ${ }^{3-6}$ Additionally, asymptomatic bacteria (ASB) places the women at risk of preterm birth and delivery of low birth weight infants. ${ }^{3,7}$

There is evidence that treating ASB in pregnancy reduces the rates of persistent bacteriuria and the women's risk of developing pyelonephritis. ${ }^{3,4,8,9}$ Based on this evidence, screening for and treating asymptomatic bacteriuria is now considered standard obstetrical care in high income countries. In resource limited countries including South Africa, the cost of standard urinary culture is a limiting factor for generalised urine screening. Therefore, other screening methods have been proposed including urine dipsticks (recommended method for antenatal screening in the public sector in South Africa), bioluminescence assays, gram staining, microscopic urinalysis and dipslide urine cultures. ${ }^{10-12}$ Dipslide urine cultures have been found to show a detection rate for asymptomatic bacteriuria comparable to that with urine culture. ${ }^{11}$ Pregnancies complicated with UTI have been well studied in high income countries, and high rates of maternal and perinatal morbidities have been reported..$^{12}$ These maternal and perinatal morbidities associated with UTI however vary from one study to another, and may be due to geographical and biological variations. A recent study performed in the
Western Cape, South Africa estimated that the prevalence of ASB in HIV-positive and HIV-negative pregnant women was $9 \%$ ( $n=$ $11)$ and $7.9 \%(n=19)$, respectively. ${ }^{13}$ An electronic literature search of studies of UTI in pregnancy performed in South Africa using the terms UTI, cystitis, pyelonephritis and ASB only revealed the Western Cape study. ${ }^{13}$ We therefore opted to carry out a retrospective review of UTI in pregnancy in a South African public hospital.

\section{Methods}

The study was conducted at a regional hospital in Durban, KwaZulu-Natal, South Africa. The study was a retrospective chart review of pregnant women admitted with the diagnosis of UTI from 1 January 2012 to 31 December 2012. Following regulatory approvals (King Edward Hospital management), including ethical permission (UKZN Biomedical Research Ethics Committee), the author obtained the names of patients admitted to the antenatal wards with an initial diagnosis of urinary tract infection and/or cystitis and/or acute pyelonephritis from the admissions register. The case records were then obtained and relevant information collected in a structured data form. The information collected included demographic data, clinical characteristics and clinical management.

Only women who had a positive urine culture were included in the study. In addition, those with positive urine cultures but with incomplete data were excluded. The exclusion criteria used to select the study cases were as follows: a negative urine culture (no growth), urine specimen unsuitable for analysis (mixed growth or leaked specimen), absence of documented urine 
culture test, multiple pregnancies and underlying renal disease (renal calculi and/or anatomical anomalies).

\section{Definitions}

Cystitis was defined as a positive urinary culture based on the detection of more than 100,000 bacteria/ml in a single voided midstream urine in a woman with symptoms of dysuria, suprapubic discomfort, urgency, frequency and in the absence of upper urinary symptomatology.

Acute pyelonephritis was diagnosed in the presence of fever, flank tenderness and symptoms of cystitis with a positive urine culture.

Maternal outcomes evaluated included:

1) Hypertensive disorders of pregnancy;

2) Acute respiratory distress syndrome (ARDS);

3) Preterm/prelabour rupture of membranes (PPROM) defined as PROM before 37 weeks of gestation;

4) Acute renal impairment (urea $>3.5 \mathrm{mmol} / \mathrm{l}$ and/or creatinine $>67 \mu \mathrm{mol} / \mathrm{l})$; and

5) Anaemia (haemoglobin $<10.5 \mathrm{~g} / \mathrm{dl}$ ).

The perinatal outcomes investigated included:

1) Gender of the foetus;

2) Perinatal death (stillbirths and early neonatal death);

3) Intrauterine growth restriction or small for gestational age (birth weight less than the 10th percentile of the birth weight distribution of the singleton deliveries in the database at each gestational age and infant sex); and,

4) Low birth weight (2500 g or lower).

The standard management of cystitis on admission in the King Edward obstetric unit when excluding preterm labour, especially when low abdominal pain was of concern, included: collection of midstream urine specimen; $\mathrm{FBC}$; urea; creatinine and electrolytes; and, commencement of empirical antimicrobial treatment with change to the other antimicrobial regimen based on the culture sensitivity. In the majority of cases, patients were discharged with follow up in the antenatal clinic; however, urine culture was not repeated for those patients who responded to treatment.

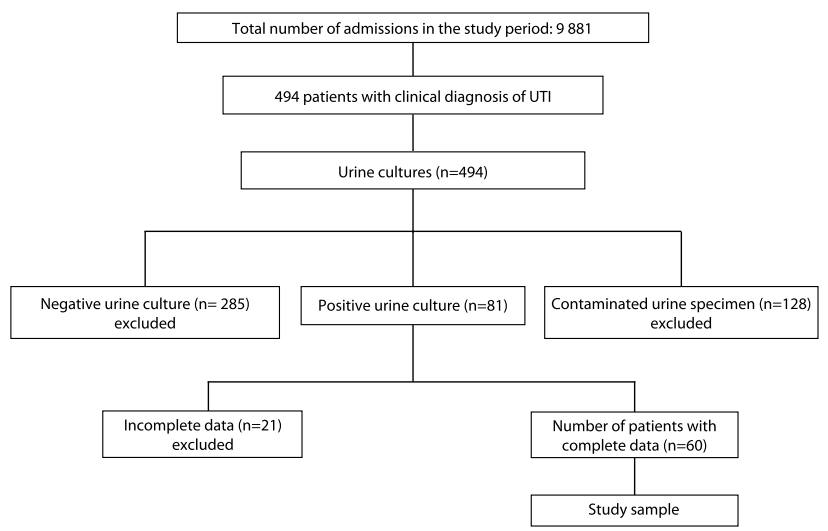

Figure 1: Study flow chart
Patients with pyelonephritis were admitted to a high care area, and empirical antimicrobial therapy was commenced. Only patients with persistent symptoms had an imaging of the urinary tract system. After discharge from hospital, follow up of all patients occurred in the antenatal clinic.

\section{Statistical analysis}

The data collected were analysed using the Statistical Package for Social Sciences (SPSS, version 21). Descriptive statistics, such as frequencies, percentages and means, was used to analyse results.

\section{Results}

Of 494 women admitted with the clinical diagnosis of UTI, 285 had a negative urine culture, 128 urine samples were contaminated and 81 had a positive culture. Amongst the 81 with a positive urine culture, 21 had incomplete data therefore 60 fulfilled the enrolment criteria and constituted the study group. Figure 1 is a diagrammatic illustration of the 494 pregnant women with a clinical diagnosis of UTI.

The admission rate for UTI was $5 \%$. The clinical characteristics of the 60 women in the study are shown in table 1.

The age group 20-35 years was predominant $(n=42,70 \%)$ and the majority were multigravida ( $n=35,58.3 \%$ ). Twenty-seven (27) of the $60(45 \%)$ were HIV-positive and $50 \%$ of these had a CD4 cell count level $<350 / \mathrm{mm}^{3}$. All women with CD4 cell count of $<350 / \mathrm{mm}^{3}(n=17)$ were on HAART (highly active antiretroviral therapy). Forty-five (45) women (75\%) complained of suprapubic pain, and 33 (55\%) complained of burning of micturition. Most women were between 28 and 32 weeks (gestational age) at admission.

Urine dipstick specimens were positive in $61.7 \%$ of cases (30 patients had leukocytes in their urine and 7 had nitrates).

Leucocytosis was found in 17 patients (32.1\%). The working diagnosis was cystitis (80\%) and pyelonephritis in the rest of the cases. E. coli was the most common pathogen isolated; and, was cultured in 24 patients (40\%). Klebsiella pneumonia was cultured in $12(20 \%)$ and Candida albicans in $6(10 \%)$.

All patients received broad spectrum antibiotics (cefradine, a first generation cephalosporine) as per local protocol. The antibiotics were changed, if required, once culture sensitivity was obtained.

The medical and obstetric conditions found in the study cases are shown in Table 2. The two cases of acute respiratory distress syndrome (ARDS) occurred in patients with pyelonephritis.

One third of deliveries occurred before 37 weeks, 5 of which were 28-33 weeks (8.3\%) and 14 were $34-36$ weeks (23.3\%).

Labour was induced in 8 cases (13.3\%) and 27 patients (45\%) were delivered by Caesarean section for obstetric indications.

Table 3 shows the gestational age and table 4 shows the neonatal outcomes. There were no stillbirths but one perinatal death occurred. The latter was a case of ventriculomegaly found on brain imaging. 
Table 1: Maternal-characteristics

\begin{tabular}{|c|c|c|}
\hline Characteristics & $(N=60)$ & Percent (\%) \\
\hline \multicolumn{3}{|l|}{ Maternal (years): } \\
\hline Mean age: 24.4 & - & - \\
\hline Range: 15-39 & 60 & 100 \\
\hline \multicolumn{3}{|l|}{ Parity: } \\
\hline Primigravida & 25 & 41.7 \\
\hline Multigravida & 35 & 58.3 \\
\hline \multicolumn{3}{|l|}{ Symptoms and signs: } \\
\hline Chronic hypertension & 2 & 3.3 \\
\hline Suprapubic pain & 45 & 75 \\
\hline Burning of micturition & 33 & 55 \\
\hline Frequency & 15 & 25 \\
\hline Renal angle tenderness & 7 & 11.7 \\
\hline Temperature & 6 & 10 \\
\hline Urgency & 1 & 17 \\
\hline
\end{tabular}

Table 2: Medical and/or obstetric complications and results of investigations

\begin{tabular}{|c|c|c|}
\hline & Cystitis & Pyelonephritis \\
\hline \multicolumn{3}{|l|}{ Medical/obstetric conditions: } \\
\hline Premature Rupture of Membranes & 5 & 0 \\
\hline Preterm delivery & 18 & 1 \\
\hline Chronic hypertension & 1 & 1 \\
\hline Acute respiratory distress & 0 & 2 \\
\hline Preeclampsia & 0 & 2 \\
\hline \multicolumn{3}{|l|}{ Results of Investigations: } \\
\hline HIV positive & 22 & 5 \\
\hline Anaemia & 17 & 9 \\
\hline "Renal dysfunction & 1 & 3 \\
\hline Repeat urine culture & 0 & 6 \\
\hline
\end{tabular}

"Transient renal dysfunction

Table 3: Gestational age at presentation and delivery

\begin{tabular}{|l|c|c|}
\hline Gestational age at initial presentation: & $(N=60)$ \\
\hline$\leq 27$ weeks & 9 \\
\hline $28-33$ weeks & 25 \\
\hline $34-36$ weeks & 81.7 \\
\hline$\geq 37$ weeks & 8 \\
\hline Gestational age at delivery: & $(N=60)$ \\
\hline $28-33$ weeks & 5 \\
\hline $34-36$ weeks & 13.3 \\
\hline$\geq 37$ weeks & 14.3 \\
\hline
\end{tabular}

\section{Discussion}

This retrospective case review shows the admission rate of UTI was $5 \%$ at the study site. This figure is low in comparison with another report in which Bacak et al (2005) report a figure of $10 \% .{ }^{12}$ The difference in figures may be due to different admission criteria and the fact that most cases of cystitis at the study site are managed on an outpatient basis. In addition, we excluded patients with incomplete documentation and in whom urine cultures were not obtained. Therefore, it is highly probable that the prevalence of UTI is higher at our study site.

Our study shows that the most common pathogen was E. coli. This is in keeping with other studies. ${ }^{4,7}$, Widmer et al also reported that $E$. coli was the most common isolate in cultures. ${ }^{13}$ What is of concern is that clinical follow up of women with UTI may have been poor because no documentation was kept on repeat 
Table 4 Neonatal outcomes

\begin{tabular}{|l|c|c|}
\hline Neonatal outcomes & Cystitis $(N=48)$ \\
\hline Perinatal mortality & $1^{*}$ & 0 \\
\hline LBW & 13 & 3 \\
\hline IUGR & 3 & 4 \\
\hline Born alive & 48 & 12 \\
\hline Stillbirths & 0 & 0 \\
\hline Mean baby weight and range & $2.7 \mathrm{~kg}(1.3-3.9)$ & $2.8 \mathrm{~kg}(1.5-4.2)$ \\
\hline
\end{tabular}

Note: LBW: Low birth weight; IUGR: Intra-uterine growth restriction

"Ventriculomegaly

cultures, as well as on any use of prophylactic urinary antimicrobials administered to prevent recurrent infections, particularly in those patients with acute pyelonephritis. Only $50 \%$ of cases of pyelonephritis had repeat cultures either during admission or at follow up. ${ }^{13}$ Standard care requires repeat urine cultures throughout the pregnancy due to the significant risk of recurrence. ${ }^{3,10,11}$ Most patients with positive cultures are asymptomatic and can be detected only with routine surveillance cultures. $3,8,11$ The inadequate clinical management of patients with UTI at the study site may be due to the large patient load, shortage of hospital beds, lack of staff, poor documentation and the fact that patients are often seen by different doctors.

The 12 cases of pyelonephritis illustrate the seriousness of this conditions as 2 of these patients required ICU care and 3 had abnormal renal function tests; similar findings have been reported. . $^{4,6} 8$

It is surprising that HIV status did not appear to play a role in the severity of the UTI. Similar findings were reported in Western Cape in South Africa by Widmer et al. ${ }^{13}$ (9\% of HIV-positive and $7.9 \%$ of HIV-negative patients had positive urine cultures). This may be due to the fact that the patients were on antiretroviral agents ${ }^{13}$

In our study, $8.3 \%$ of the women with UTI went on to develop preterm rupture of membranes (8.3\%). Similar figures have been reported in women with PROM..$^{3,6,8}$ It has been demonstrated that bacteriuric women may develop an amniotic fluid infection with the same organisms commonly isolated from urine specimens such as E. coli. ${ }^{14}$ Bacterial collagenase may weaken the foetal membranes and predispose them to rupturing, which subsequently leads to the onset of labour. ${ }^{14}$

With regard to preterm deliveries, the result of this study (31.6\%) is consistent with the finding of previous studies. ${ }^{3-5,15}$ Surprisingly, preterm deliveries in most cases occurred in women with cystitis. This is in contrast with Mazor-Dray et al., whose findings showed an association between a higher proportion of preterm birth and the severity of UTI. ${ }^{6}$ This difference may be due to the recurrence of UTI after treatment as urine cultures were not repeated in many instances in our study.

Anaemia was present in $44.8 \%$ of the women with UTI. Maternal anaemia has been reported in $25 \%$ to $66 \%$ of antepartum patients with pyelonephritis. ${ }^{16}$ The most probable cause of this haematological change is haemolysis secondary to endotoxin. ${ }^{16}$ However, other contributory factors for anaemia (low socioeconomic factors, chronic diseases e.g. HIV, etc.) cannot be excluded in our study. Anaemia in pregnancy is a global health problem, and higher rates are reported from poor countries, therefore we can draw no definite conclusions on the prevalence of anaemia and UTI. ${ }^{17}$

In this study $25.9 \%$ of women had contaminated urine specimens. Bekeris et al., found the median institution rate of contamination to be $15 \% .^{18}$ This high urine contamination rate is probably related to post-collection processing of specimens as it is common to find specimens lying uncollected for hours in the collection box before transport to the laboratory.

\section{Limitations}

Our study does have limitations in that it is retrospective in nature. There was also poor documentation of results from case files and hospital information, which is essential for clinical audits. However, this descriptive study highlights the need to improve the quality of care of antenatal patients who have UTI.

\section{Recommendations}

We suggest the following recommendations to health caregivers:

Patient records should be up to date and relevant information documented.

- All pregnant women with UTI should have a repeat urine cultures after completion of the treatment to detect and treat cases of recurrence.

- The handling of urine specimens should improve with appropriate handling and timeous processing of urine specimen after collection.

We suggest the following recommendations to healthcare policy makers:

- Introduction of urinary culture for screening for ASB in pregnancy should be a part of the routine antenatal care.

We suggest the following recommendations to the scientific community:

- Better designed prospective studies, as well as studied based on larger populations, are needed to evaluate the impact of UTI in pregnant women in our setting.

\section{References}

1. Wing DA, Fasset MJ, Getahun D. Acute pyelonephritis in pregnant : an 18-year retrospective analysis. Am J Obstet Gynecol. 2014 [cited 2015 Aug 31];2010(3):219. E1-6. doi:10.1016/j.ajog2013.10.006. Epub 2013 Oct 5.

2. Sheffield JS, Cunningham FG. Urinary tract infection in women. Obstet Gynecol. 2005;106:1085-1092.

3. Matuszkiewiez-Rowinska J, Malyszko J, Wieliczko M. Urinary tract infections in pregnant: old and unresolved diagnostic and 
therapeutic problems. Arch Med Sci. 2015;11(1):67-77. doi:10.5114/ aoms.2013.39202

4. Farkash $E$, Weintraub $A Y$, Sergienko $R$, et al. Acute antepartum pyelonephritis in pregnant: a critical analysis of risk factors and outcomes. Eur J Obstet Gynecol Reprod Biol. 2012;162:24-7.

5. Schneeberger C, Geerlings SE, Middleton $P$, et al. Interventions for preventing recurrent urinary tract infection during pregnant. Cochrane Database Syst Rev. 2015;7.CD009279. doi:10.1002/14651858. CD0092/9.pubs

6. Mazor-Dray E, Levy A, Schlaeffer E et al. Maternal urinary tract infection : is it independently associated with adverse pregnant outcome? J Matern Fetal Neonatal Med. 20090;22:124-32.

7. Oladeinde $\mathrm{BH}$, Omoregie $\mathrm{R}$, Oladeinde $\mathrm{OB}$. Asymptomatic urinary tract infections among pregnant women receiving antenatal care in a traditional birth home in Benin City, Nigeria. Ethiop J Health Sci. 2015;25(1):3-8.

8. MacLean $A B$. Urinary tract infection in pregnant. Int J Antimicrobial Agents. 2001;17(4): 273-76.

9. Smaill FM, Vazquez JC. Antibiotics for asymptomatic bacteriuria in pregnant. Cochrane Database Sys Rev. 2015 [cited 2015 Aug 30];8 (Epub ahead of print): CD000490.

10. Guidelines for maternity care in South Africa 2007: a manual for clinics, community health centers and district hospitals. 3rd edition. National Department of Health, Pretoria. 2007, pp 27.

11. Mignini L, Carroli G, Abalos E, et al. For the World Health Organization Asymptomatic Bacteriuria. Accuracy of Diagnostic Tests to Detect
Asymptomatic Bacteriuria During Pregnancy. Am Coll Obstet Gynecol. 2009;113(2):112-5.

12. Bacak SJ, Callaghan WM, Dietz PM, et al. Pregnancy-associated hospitalization in United States, 1999-2000. Am J Obstet Gynecol. 2005;192:592-97.

13. Widmer TA, Theron G, Grove D. Prevalence and risks of asymptomatic bacteriuria among HIV positive pregnant women. S Afr J Epidemiol Inf. 2010;25(1):28-32.

14. Banhidy F, Acs N, Puho EH, et al. Pregnancy complications and birth outcomes of pregnant women with urinary tract infections and related drug treatments. Scand J Infect Dis. 2007;39:390-397.

15. Dawkins JC, Fletcher HM, Rattray CA, et al. Acute pyelonephritis in pregnant. A retrospective descriptie hospital-based study. ISRN obstet Gynecol. 2012 [cited 2015 Aug 31]: 519321 online 2012, Nov 14, doi:10.5402.2012/519321

16. Gomi H, Goto Y, Laopaiboon M, et al. Routine blood investigations in the management of pyelonephritis in pregnant. Cochrane Database Rev. 2015 Feb 13; 2: CD009216. doi:10.1002/14651858.CD009216. pub2

17. Pena-Rosas JP, DeRegil LM, Dowswell T, et al. Intermittent oral supplementation during pregnant. Cochrnae Database Sys Rev. 2013 July 11;7(CD00999): 7. doi:10.1002/14651858.

18. Bekeris LG, Jones BA, Walsh MK, et al. Urine culture contamination: a college of American Pathologists Q-Probes study of 127 laboratories. Arch Pathol Lab Med. 2008;132(6):913-7. 